\title{
Profesionalización de LA PSicología Mexicana. Un Estudio SOBRE LOS EGRESADOS DE EDUCACIÓN A DISTANCIA
}

\section{Omar Moreno Almazán UNAM FES IZTACALA}

\section{MÉXICO}

\section{RESUMEN}

El estudio de la práctica profesional a partir del egreso e incorporación laboral, nos brinda elementos para comprender la funcionalidad y los problemas relacionados con los planes de estudio y la demanda laboral. El presente trabajo tiene por objetivo describir indicadores presentes en egresados en psicología del sistema a distancia (SUAED) de la UNAM. Su desarrollo ha sido a partir de una investigación exploratoria en la cual se hizo un contacto directo con una muestra de ellos a fin de conseguir información a través de herramientas digitales. Destacan elementos importantes sobre todo en las fortalezas que esta modalidad ha brindado y de las cuales han permitido una incorporación laboral exitosa, pero a su vez también salen a la luz algunos indicadores sobre las debilidades formativas que tienen impacto una vez que se encuentran en el ejercicio profesional. Aún es posible discutir sobre la tendencia de estos resultados.

Palabras Clave: Egresados, evaluación, educación a distancia, psicología.

\section{Professionalism OF MeXICAN PSYCHOLOgY. A STUDY OF GRADUATES OF DISTANCE EDUCATION}

\section{ABSTRACT}

For a long time studies have been developed that allow us a glimpse of the professionalism and training of psychology within universities and the performance of its graduates in the workplace; is negligible however the presence of indicators to explore the conditions that people who graduate degree in educational psychology from a distance mode, of which much has been speculated about the possibility, usefulness and relevance in our country live. The present study is one of the first researches with psychology graduates in a distance system (SUAED UNAM). Its development has been based on a descriptive research in which direct contact was made with a sample of them in order to get information through digital tools. Highlight important elements especially on the strengths that this method has provided and which have enabled successful work integration, but in turn also brought to light some pointers on training weaknesses that impact once they are in practice. It is still possible to discuss the trend of these results.

Keywords: Graduates, assessment, distance education, psychology.

Moreno, A. O. (2015). Profesionalización de la Psicología: Un estudio sobre los egresados de educación a distancia. Revista Digital Internacional de Psicología y Ciencia Social, 1(1), 50-61. 


\section{BITÁCORA DEL ARTíCULO}

PROFESIONALIZACIÓN DE LA PSICOLOGÍA MEXICANA. UN ESTUDIO SOBRE LOS EGRESADOS DE EDUCACIÓN A DISTANCIA

Omar Moreno Almazán

\section{Bitácora del Artículo:}

Recibido: 6 de octubre de 2014

Aceptado: 28 de enero de 2015

Publicado en línea: 7 de febrero de 2015

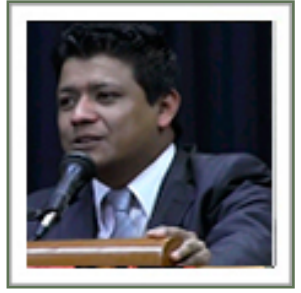

Omar Moreno Almazán

UNAM - FES Iztacala

Correo: almazanomar@gmail.com

Doctor en Psicología, en el área de Psicología Educativa y del Desarrollo por la UNAM, 2015; Licenciado en Psicología por la FES Iztacala-UNAM. Profesor de la Carrera de Psicología en Sistema de Universidad Abierta y Educación a Distancia, UNAM.

Ver más...

\section{Datos de filiación del autor}

Carrera de Psicología en Sistema de Universidad Abierta y Educación a Distancia, FES Iztacala, UNAM 


\section{TABLA DE CONTENIDO}

MÉTOdo

55

Participantes, 55

Procedimiento, 55

Mediciones, 55

Análisis estadísticos, 55

Resultados

Datos generales, 55

Trayecto académico, 57

Valoración hacia la carrera, 58

CONCLUSIONES

REFERENCIAS

MetA-AnÁlisis del Artículo

Dimensión Cuantitativa, 63

Dimensión Cualitativa, 65

IR A LA Historia del Proceso Editorial 
Introducción

A nivel mundial se ha hecho énfasis en las formas en las que las instituciones educativas de nivel superior evalúan los procesos de planeación, gestión y cambio institucional. Evidentemente los países desarrollados son los que han impulsado estos procesos desde hace algunas décadas y que, en este tenor México se incorporó a ello a mediados de los ochenta. Para entonces la formación de la psicología tenía una tendencia prominente en enfoques conductistas, seguidos de enfoques relacionados con la psicología transcultural, los modelos psiquiátricos-psicométricos, y en menor medida los movimientos en psicología social y en psicología cognitiva; los cuales todos dieron trayecto a muchas escuelas presentes en un modelo educativo tradicional y presencial, con algunas pocas en los modelos de educación abierta. En esta misma línea, no fue sino hasta el 2005 que se abre en nuestro país el modelo de educación a distancia, apoyado en las tecnologías de la informática y comunicación.

El escenario en el que la formación de la psicología mexicana se mantuvo hasta finales del siglo XX, contaba ya con la experiencia de los cambios de paradigmas ideológicos derivados de acontecimientos sociales relevantes como el sismo de 1985; para entonces la diversificación en la enseñanza y consolidación de la psicología brinda importancia a esta diversidad que se manifiesta en su actuar. Muestra de ello se ve en el desarrollo de la productividad que tanto los recién egresados como los psicólogos en función, juntamente con los académicos, desarrollaron.
Para el caso de la realización de eventos académicos en donde la participación de los psicólogos que se enfrentaron a la situación social, se pueden contar desde la creación y desarrollo de eventos académicos tales como los coloquios profesionalizantes que se hicieron principalmente en la ENEP Zaragoza de la UNAM a partir de 1987 y en los congresos "Al encuentro de la Psicología Mexicana" organizados principalmente a partir de 1990 (Véase Tabla 1).

El desarrollo de estas inciativas han permitido un intercambio en el proceso de formación y de actualización de los recién egresados en psicología en el sentido de que se cristaliza o consolida su conocimiento en el sentido de su inclusión social a En partir de la expansión de la investigación y del el 2005 se impartió la primer licenciatura en línea intercambio de los distintos puntos de vista de los cada vez más números psicólogos en ejercicio profesional (Galindo, 2004).

Otro referente importante se sitúa en los trabajos escritos, donde en la historia de la psicología de nuestro país tiende a existir una situación especial, pues la iniciativa de crear y consolidar revistas especializadas ha estado presente desde la segunda mitad del siglo $X X$, sin embargo su desarrollo ha sido decreciente. El panorama que existió en México para entonces se puede reflejar con el número de publicaciones especializadas en psicología y con impacto nacional y que se puede ver en la Tabla 2.

Es necesario mencionar que la presencia de la investigación, difusión y consolidación de la psicología en México, no es un asunto meramente documentado, pero del cual existen datos sólidos. En una recopilación realizada por Díaz, Cruz Pérez, Cruz García, Aguilar y

Tabla 1 .

Lugar de creación del Congreso "Al Encuentro de la Psicología Mexicana"

\begin{tabular}{|l|c|l|}
\hline S & AÑo & LUGAR \\
\hline I & 1990 & Tlalnepantla,E.de M. (UNAM:ENEP-IZTACALA) \\
\hline II & 1992 & Tlalnepantla,E.de M. (UNAM:ENEP-IZTACALA) \\
\hline III & 1995 & México D.F. \\
\hline IV & 1998 & Toluca, E. de M. \\
\hline VI & $2000^{*}$ & Guanajuato, Gto. \\
\hline VI & 2002 & Puebla, Pue. \\
\hline
\end{tabular}


Tabla 2 .

Revistas especializadas en Psicología en México

\begin{tabular}{|c|c|c|}
\hline Década & $\begin{array}{l}\text { Número de } \\
\text { revistas }\end{array}$ & $\begin{array}{c}\text { Revistas que desaparecen o se vuelven } \\
\text { irregulares }\end{array}$ \\
\hline 50 & 1 & 0 \\
\hline 60 & 2 & 0 \\
\hline 70 & 9 & 1 \\
\hline 80 & 17 & 4 \\
\hline
\end{tabular}

Vargas (2010) es posible mencionar que para el año 2010 existían en nuestro país cerca de 351 universidades e institutos tanto públicos como particulares, que ofrecen la licenciatura en psicología (número que creció considerablemente a inicios de 1990 donde se tenía un registro de 66 escuelas). De este conjunto, la Universidad Nacional Autónoma de México (UNAM) ha sido hasta entonces la institución que determina en gran medida la investigación y actividad formativa tanto en México como en Iberoamérica, lo cual le brinda una responsabilidad social muy importante en el momento de consolidar a sus egresados.

Ante este panorama, fue en 2005 que funcionó la primera licenciatura en psicología impartida en línea, la cual se creó por la UNAM campus Iztacala con una matrícula de 61 alumnos y que ha ido en crecimiento hasta llegar a su cifra que, para el año 2013 reportó una matrícula de 2010 alumnos inscritos, es decir, en 10 años la demanda de esta licenciatura en la modalidad en línea creció en una proporción de 3295\% de su etapa inicial.

Otro aspecto importante a considerar es la condición que mantienen los egresados en psicología, a partir de la importancia que existe no solo en intentar responder a la demanda formativa, sino también en exponer la situación a la cual se enfrentan las personas que ingresan a esta profesión. Pocos son los estudios que existen sobre el seguimiento de egresados en psicología de nuestro país, además de que la actualización de estos mismos datos tiende a mermarse a partir del surgimiento de modificaciones a planes y programas de estudio. Con el fin de exponer algunos indicadores, se retoman algunos casos.

En un seguimiento realizado por Macía y Pineda (1998) sobre la situación de 180 egresados en psicología, de la UNAM campus Iztacala en modalidad presencial, encontraron lo siguiente:

- $\quad$ El $80 \%$ de ellos trabajaba, de los cuales el $31.7 \%$ se dedicaba al sector público, $30 \%$ al sector privado y $13.3 \%$ a empresa particular.

- $20 \%$ de ellos ocupaba mandos medios, otro $20 \%$ ocupaba puestos de empleados o auxiliares y $16.7 \%$ eran directivos.

- La ocupación primordial se enfocó a la psicología clínica, educativa y metodológica.

- La demanda ocupacional a la que se enfrentaron correspondió a actualización en psicología organizacional y psicometría; mientras lo que les resultó en menor demanda fue la psicología experimental de laboratorio.

- A pesar de considerar su conocimiento teórico, metodológico y aplicado como bueno, el 55\% de ellos reportó como regular su conocimiento ante las exigencias sociales a las que se enfrentaron.

Otro estudio realizado por la Universidad Autónoma Metropolitana, unidad Iztapalapa (2008) se levantó un seguimiento a 113 egresados de la carrera de psicología social y en el cual hallaron lo siguiente para el Plan de Estudios de 2003:

- El $77.19 \%$ de ellos se encontraba laborando, de los cuales el $95.5 \%$ tenía un nivel de empleado y $4.5 \%$ era propietario. Destaca que ninguno reportó trabajar de manera independiente.

- El $50 \%$ trabajaba en el sector público, $47.7 \%$ lo hace en sector privado y el resto en asociaciones o sindicatos.

- El $41.9 \%$ reporta coincidencia entre lo que aprendieron en su plan de estudios y lo que enfrentan en su mercado laboral.

- Su desempeño se realiza principalmente en el sector educativo ( $60 \%$ de ellos), siendo el $30 \%$ de ellos dedicados a la docencia. 
- Las principales dificultades para conseguir empleo han sido la escasa experiencia (28.6\%) y que no hay suficientes oportunidades de empleo $(28.6 \%)$

- Los principales elementos que facilitaron la obtención de empleo fue, además de las habilidades mostradas en las entrevistas, el contar con el título profesional (66\% de coincidencia) y contar con estudios extracurricualres como en idiomas, paquetería en cómputo, conocimientos especializados (60.4\%)

- $60.4 \%$ de ellos manifestó que la enseñanza metodológica fue la que coincidió más en su inclusión al campo laboral, mientras que las prácticas de laboratorio no les apoyaron en ningún momento.

- Las habilidades que su plan de estudios les brindó, además de los conocimientos básicos en psicología, fueron las habilidades para la búsqueda de información a través de herramientas tecnológicas (48.3\%) y la capacidad de pensamiento analítico y pensamiento creativo (34\% para cada uno)

Con base a estos elementos, es posible observar la coincidencia que existe en varios elementos de carácter formativo, sin embargo los datos corresponden a un momento en el que la psicología seguía fuertemente regida por modalidades educativas presenciales, sin que exista una base que permita hacer un comparativo con la situación que actualmente se refleja.

Ante este panorama, el propósito del presente estudio ha sido describir las condiciones del ejercicio profesional que mantienen los egresados en la carrera de psicología, adscritos al Sistema de Universidad Abierta y Educación a Distancia (SUAED), de la UNAM campus Iztacala, a partir de la exploración de su trayecto laboral y el impacto de la formación recibida durante su licenciatura.

\section{Método}

\section{Participantes}

Se consideró a un total de 34 exalumnos que contaron con el $100 \%$ de créditos, egresados de la carrera de psicología en el SUAED. El tipo de muestra fue no probabilístico.

\section{Procedimiento}

Se envió el cuestionario vía correo electrónico a un conjunto de 50 egresados, a quienes se les invitó a participar respondiendo al cuestionario y enviándolo resuelto.

\section{Mediciones}

Para este estudio, se realizó un cuestionario de seguimiento de egresados. El cuestionario fue de tipo Likert con un total de 40 ítems. Las dimensiones estudiadas fueron:

Trayecto Académico, Trayecto Profesional, Trayecto Laboral y Valoración hacia la carrera.

Para fines del presente estudio, se consideraron las dimensiones 1 y 4 , a saber:

Trayecto Académico: Corresponde a la obtención de datos que describen su inclusión y permanencia en el plan de estudios de psicología SUAED hasta su egreso, incluyendo las condiciones que enfrentaron en el proceso de egreso y titulación.

Valoración hacia la carrera: Corresponde a la valoración que los participantes han dado a las características curriculares y formativas de la licenciatura, en función a su utilidad, actualización y funcionalidad en el momento de su inclusión laboral.

\section{Análisis estadísticos}

La base estadística se sitúa principalmente en análisis de frecuencia y métodos de análisis descriptivo, a partir de que son los procedimientos estadísticos que nos permiten una descripción específica de los fenómenos comportamentales.

\section{Resultados}

Se presentan los datos descriptivos a partir del siguiente orden: a) Datos generales, b) Trayecto Académico y c) Valoración hacia la carrera. Los resultados son los siguientes:

\section{Datos generales}

Participaron en este estudio egresados del SUAED Psicología que actualmente reportan su ubicación como lo muestra la figura 1.

Encontramos una mayor inclusión en estados en donde el sistema a distancia comenzó a incidir a nivel nacional, sin embargo resulta importante señalar que la presencia 


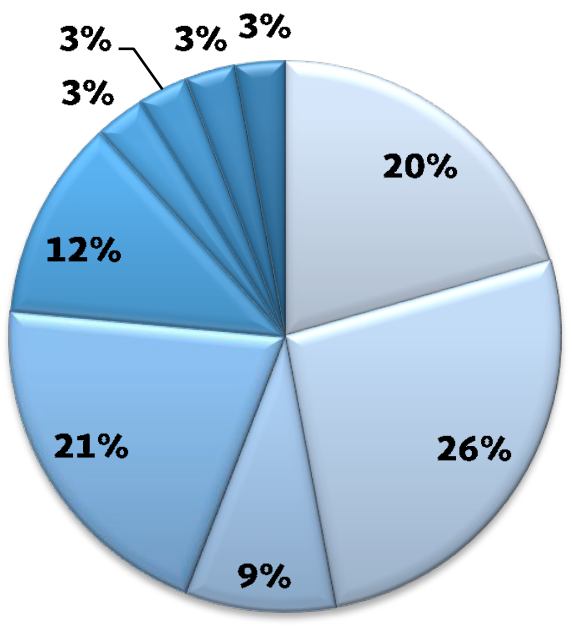

$\square$ Tlaxcala
$\square$ Oaxaca
$\square$ Hidalgo
$\square$ Estado de México
$\square$ DF
$\square$ Puebla
$\square$ Guanajuato
$\square$ Durango
$\square$ San Luís Potosí

Figura 1.

Entidad de vivienda actual

de egresados oriundos del Valle de México es creciente, aún a pesar de que éstos últimos contarían con mayor posibilidad de participar en la formación presencial en la misma carrera e institución. La proporción en cuanto a sexo se presenta en la Figura 2.

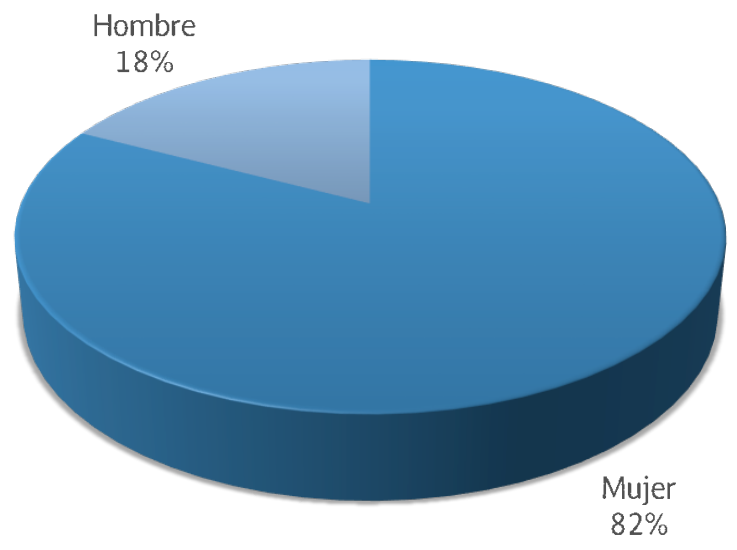

๑Mujer $\square$ Hombre

Figura 2.

Sexo de los participantes

La edad promedio de los egresados es de 36.9 años de edad, habiendo profesionistas que van desde los $24 \mathrm{y}$ hasta los 56 años de edad.

De ellos, destaca además la condición civil por la que se encuentran en este momento (Véase Tabla 3).

Mientras que la condición en cuanto al número de hijos se puede observar en la figura 3.
Los datos que presentan los egresados de esta modalidad, resultan ser más variados en relación los que se encuentran en sistemas presenciales. Como muestra está el comparativo que hay en egresados de la UAM del sistema presencial donde casi el $64 \%$ son solteros sin hijos (Véase UAM, 2008).

Tabla 3 .

Estado Civil

\begin{tabular}{|c|c|}
\hline Estado Civil & N \\
\hline Soltero & 9 \\
\hline Casado & 13 \\
\hline Unión libre & 1 \\
\hline Separado o divorciado & 10 \\
\hline Madre soltera & 1 \\
\hline
\end{tabular}

Este panorama nos brinda una información general de las condiciones sociales que los egresados en psicología del SUAED mantienen en este momento. Vale decir que dicha condición corresponde a las misiones originales del sistema, que se manifestaron desde la creación del SUA, con la encomienda de brindar una formación universitaria a sectores de la población que carece de las mismas oportunidades sociales, geográficas o ideológicas para cursar estudios profesionales, sin perder la encomienda universitaria de mantener los principios que regulan la actuación institucional. 
No tengo $\quad 1 \quad 2$ a $3 \square 4$ a $5 \square$ Más de 5

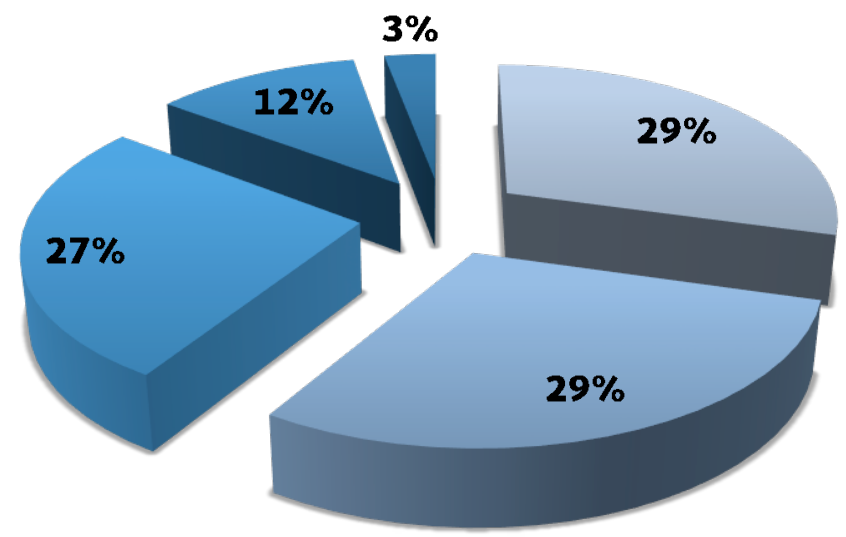

Figura 3.

Número de hijos

\section{Trayecto académico}

La licenciatura en psicología del SUAED cuenta con un plan de estudios creado ex profeso para esta modalidad, mismo que fue aprobado en 2004 y entre sus particularidades destaca la posibilidad de cursar la licenciatura en un periodo $100 \%$ superior al tiempo establecido, es decir, que el plan de estudios está creado para cursar los estudios universitarios en 9 semestres, habiendo la posibilidad de concluir dichos estudios en un periodo de hasta 18 semestres, justamente por las particularidades de esta población. En este sentido, se está reportando que los egresados invirtieron justamente los 9 semestres para terminar sucarrera; habiendo casos que reportan haber invertido hasta 6 años en ello y quienes incluso concluyeron en periodo de 3 años y medio. El promedio académico que obtienen al final es de 9.14, con casos que van desde 8.5 y hasta 9.81 en su promedio final.

Estos indicadores nos señalan que quienes están dentro de esta licenciatura no están utilizando todo el tiempo que la misma estructura curricular les brinda, incluso tienen la posibilidad de terminar antes del tiempo normal y además lo hacen con alta calidad formativa, siendo que hasta este momento el mínimo en el promedio de rendimiento es de un nivel suficiente para acceder a cualquier formación en posgrado o ser considerado un alumno de buen nivel, de acuerdo a las concepciones que las instituciones han otorgado.

Pero esta condición por sí misma es aún más entendible a partir de señalar que el $56.8 \%$ de ellos reporta haber trabajado mientras estudió, 32.4\% reporta haber trabajado al menos alguna vez y solo el
2.7\% dedicó su tiempo completo al estudio, sin tener la necesidad absoluta de dividir su tiempo trabajando, lo cual tampoco los exenta de una condición absoluta a saber que la mayor parte de ellos cuenta con responsabilidades familiares y sociales distintas a las que son comunes observar en alumnos jóvenes escolarizados.

Destaca además que su trayecto académico también es variado en cuanto a sus antecedentes formativos académica reportada, se puede observar en la figura 4 la variabilidad de sus condiciones.

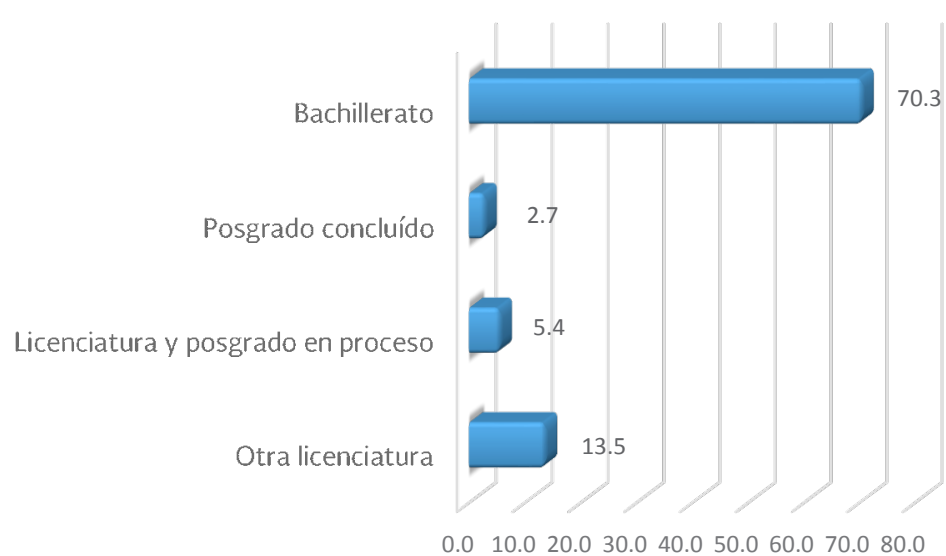

Figura 4.

Proporción observada en estudios previos

Evidentemente esta modalidad está encauzada no solo a personas que cuentan con estudios de bachillerato, quienes además no resulta difícil determinar que dichos estudios no han sido recientes en comparación con los presenciales; sino que además se cuenta 
en la matrícula con personas que cuentan con otras licenciaturas e incluso con una formación en posgrado que puede ser de distintas áreas. Esta condición dispone de formas de asimilación de su carrera en una manera diferente a la cotidianamente observada.

\section{Valoración hacia la carrera}

Ante estas características resulta importante conocer ¿Cuál es la situación a la que nuestros egresados se enfrentan? Pero sobretodo, ¿Qué tanto les resulta útil esta formación recibida? Para el caso de esta muestra disponemos de algunos datos.

Se reporta que actualmente el $89.2 \%$ de los egresados está trabajando, habiendo por ende un $10.8 \%$ que por alguna razón no está ejerciendo la carrera a pesar de ser egresado, situación que le brinda la condición de titulado de acuerdo con el plan de estudios del SUAED.

Entre los problemas que han enfrentado al momento de conseguir empleo, destaca lo observado en la tabla 4. Las condiciones de la no obtención de empleo o en la búsqueda que se problematiza a partir de padecer situaciones comunes con el resto de egresados.

Tabla 4.

Problemas principales en la obtención de empleo

\begin{tabular}{|c|c|}
\hline Razones & N \\
\hline No he buscado trabajo & 2 \\
\hline Los salarios son bajos & 5 \\
\hline Se prefieren egresados de otras universidades & 13 \\
\hline Se prefieren egresados de otras modalidades & 1 \\
\hline Me dedico a negocio personal & 5 \\
\hline Otro & 4 \\
\hline
\end{tabular}

En este sentido es necesario remarcar que el hecho de ser egresados de un sistema a distancia no es razón para la no obtención de empleo y que la razón principal es atribuible más bien a la presencia de salarios bajos, situación que se vive en todo el país y que es ajena a las características de los planes de estudio. Una situación interesante se presenta en quienes se dedican a negocio particular, siendo o no de esta profesión; pero que habla de habilidades de emprendimiento que puede resultar una fuerte alternativa de autoempleo en esta profesión.

¿En que se emplean nuestros egresados? Es también una pregunta valiosa en el sentido de que el plan de estudios del SUAED brinda la opción de adquirir conocimientos específicos de profesionalización, habiendo aún con ello, la opción de egresar como
Otra Clínica Educativa $\square$ NEE $\square$ Salud Organizacional

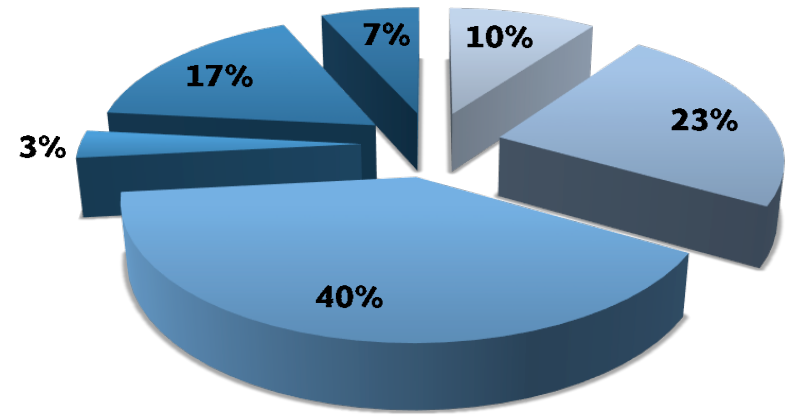

Fígura 5 .

Áreas de empleo

licenciados en psicología de manera general. En la figura 5 es posible observar las áreas de mayor ejercicio profesional de nuestros egresados.

La tendencia corresponde mucho a la presente en los estudios de carreras presenciales, siendo sobresaliente que la psicología educativa parece brindar más opciones de empleo, seguido de la psicología clínica y la del campo de la salud. De estas áreas de trabajo, destaca el perfil de puesto que nuestros egresados están presentando y que pueden ser observados en la figura 6.

La posibilidad que brinda este plan de estudios y esta modalidad, parece ampliar en cierta medida la oportunidad de emplearse en distintos niveles, en el hecho de que el índice observado tanto en el trabajo independientecomoen lospuestos demandoodirección, son más altos que los presentados en otrosinformes de seguimiento de egreso. Aún con ello, destaca la condición de que la mayor parte de los egresados se ubican en una función operativa del lugar de trabajo.

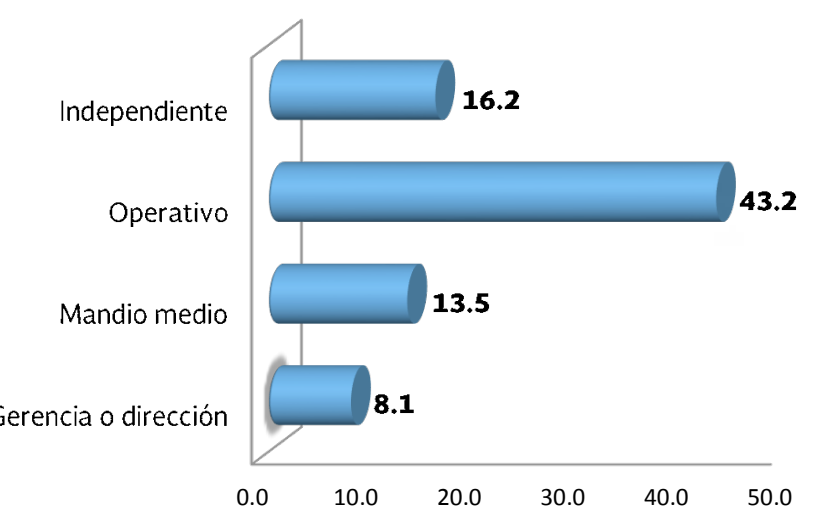

Figura 6.

Proporción en ubicación de puesto de empleo 


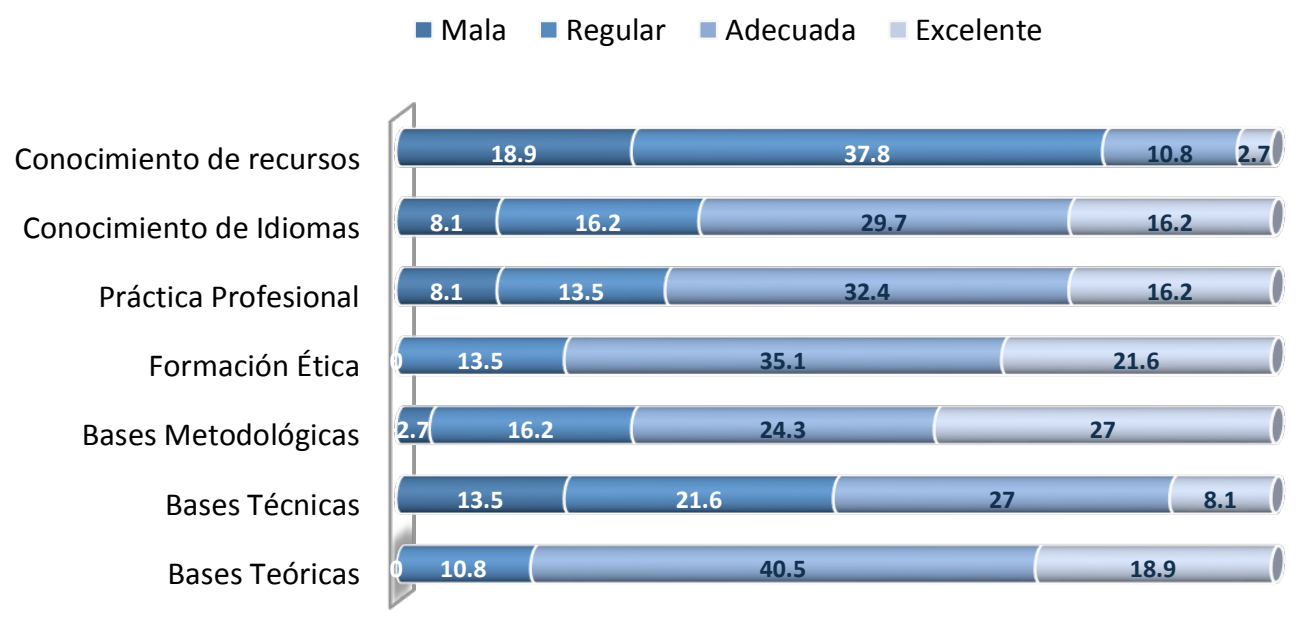

Figura 7 .

Valoración estimada a partir de la demanda laboral

En función a ello, se determinó también la lógica de su plan de estudios y lo que actualmente enfrentan en el campo profesional, pidiendo que valoraran algunas dimensiones relacionadas con la demanda laboral. Los resultados se presentan en la figura 7.

Como se puede observar, parece que el plan de estudios es adecuado a las demandas que ellos enfrentan en su condición de egresados, siendo principalmente en las áreas de formación teórica, en la cual ellos conocen sobre las bases históricas y conceptuales de los preceptos que rigen a la psicología; en la formación ética como elemento que rige la aplicación de conocimientos en beneficio de la población y la de prácticas profesionales, dando una descripción de que el plan de estudios hace una inclusión al campo de aplicación casi desde sus inicios y esto les permite una mejor adaptación al sistema. De manera sobresaliente además parece que el conocimiento de bases metodológicas resulta ser el área de mayor impacto y formación de los egresados del SUAED, en la cual ellos adquieren los conocimientos y habilidades para desarrollar sus prácticas y sus procedimientos de actuación.

Sin embargo ellos también manifiestan la debilidad formativa del plan de estudios en áreas tales como la de Bases Técnicas y en la cual ellos adquieren conocimientos de estrategias específicas de acción, tales como estrategias particulares ante casos específicos, técnicas terapéuticas o de evaluación; estrategias docentes o procedimientos particulares de reclutamiento y selección, como podrían ser algunos ejemplos; reconociendo además que estos conocimientos los adquirieron en la difusión y asistencia de eventos académicos específicos tales como coloquios, congresos o foros especializados, del cual un punto óptimo es la inclusión de estas actividades como parte de sus condiciones de permanencia y egreso en este plan de estudios.

En una forma más amplia, se reconoce la debilidad más fuerte en el conocimiento de recursos, donde ellos asumen que el plan de estudios no les brinda los recursos formativos suficientes para ejercer su práctica profesional tal y como se demanda en el exterior y que se trata de conocimientos tales como el uso de pruebas psicométricas o proyectivas, de paquetería básica en software especializados o herramientas de trabajo psicológico, dando una impresión de que saben crear metodologías de trabajo e investigación pero carecen de los elementos con los que puedan llevarlas a cabo con eficacia. Este se convierte en la debilidad más importante del sistema SUAED Psicología.

Destaca el hecho de que el $90 \%$ de ellos considera de suma importancia que el sistema mantenga la promoción de cursos, eventos académicos y procesos de actualización con la idea de que se vuelvan métodos de aprendizaje continuo, virtuales y apegados a la realidad profesional a la que se enfrentan. Llama la atención que, a diferencia de otras carreras, aquí no se solicita como tal la inclusión de bolsas universitarias de trabajo y se motivan más por el desarrollo de actividades conjuntas y de identidad universitaria.

\section{Conclusiones}

¿Cuál es la necesidad conceptual y formativa de crear seguimientos de egresados? En realidad la utilidad es importante si consideramos los factores de evaluación 
y toma de decisiones que se pueden hacer para mejorar los procesos formativos. Autores como Peña (2009) señalan que la importancia radica en conocer las tendencias de crecimiento, las vivencias de los nuevos profesionistas y el conocimiento de sus experiencias profesionales; pero de manera particular es posible señalar que en el caso de carreras que se imparten a través de sistemas de telecomunicación, de las cuales entran en una modalidad denominada "a distancia", han tenido una escepticismo histórico en nuestro país, lo cual hizo que en su momento se convirtiera en especulación pero que ahora se puede resolver en formas tangibles de estudio e investigación, ampliando con ello el panorama general de la formación psicológica.

De manera precisa, la participación de los egresados en este estudio se ha visto motivada por el interés que ellos mismos demuestran sobre las condiciones que guarda su sistema, en el hecho de que ellos mismos se saben parte de la historia educativa de la psicología en una generación nueva, donde las tecnologías juegan un papel importante como en otras partes del mundo, pero que en México había sido hasta hace poco menos de una década, un mito.

Una primer conclusión puede ser que esta clase de investigaciones puede servir para la mejora y la toma de decisiones curriculares en sistemas de formación a distancia, básicamente en las relacionadas con la formación en psicología, con el fin de evitar el estatus pasivo a nivel curricular y buscar nuevas formas de mejora e incorporación de elementos formativos que podrían brindar mayores habilidades y conocimientos a los egresados. En algunos planes curriculares, sobre todo a nivel de posgrado, se cuenta con sistemas de seguimiento que permiten la mejora continua y la diversificación de oferta formativa a partir de la valoración de los mismos estudiantes, además de tomar sus opiniones como parte de los indicadores de calidad institucional.

De forma específica, un punto de análisis sobre estos resultados nos hacen coincidir con la idea expresada en otros estudios antecedentes, correspondientes a sistemas presenciales y que nos permiten plantear ¿Qué necesitan y qué no necesitan los estudiantes en un sistema formativo? Esto de manera independiente a la modalidad que puedan tener. Al parecer ellos requieren formación no solo teórica o metodológica, sino que además el conocimiento de estrategias, procesos, instrumentos y recursos. Este estudio coincide con los desarrollados por la UAM (2008) y por Macía y Pineda (1998) en donde los egresados sugieren no solo mantener, sino ampliar los contenidos y enseñanzas relacionadas con la adquisición de habilidades específicas, conocimientos técnicos y el uso de recursos especializados, expuestos en conocimiento teórico, metodológico, técnico, computacional, técnico y estadístico; particularizando en este sector, el empleo que las instituciones pueden desarrollar para la creación de modelos virtuales de enseñanza y actualización, con el fin de contrarrestar las barreras geográficas o temporales a las que están sujetos. La utilidad del conocimiento basado en preceptos experimentales es quizá el menos favorecido en la preferencia y funcionalidad de la acción profesional en psicología.

Esta afirmación adquiere una particular importancia a partir de conocer los perfiles con los cuales están egresando: Son personas que en promedio son mayores a los 30 años de edad, con responsabilidades sociales diversas tanto en su empleo, su formación académica antecedente, su ubicación geográfica y su estado civil; con ello nos queda claro que estas condiciones no han sido un impedimento para que puedan ingresar a un proceso formativo universitario, sino que además lo hacen con altos estándares en cuanto a su promedio, con el compromiso de hallar formas de profesionalización extracurriculares y logrando posicionarse en el campo laboral casi de manera inmediata.

Conviene recordar con ello que los perfiles que se pretenden en esta modalidad, corresponde al desarrollo de habilidades autorregulatorias de aprendizaje; por lo tanto, aquellos que han egresado adquieren en su andar formativo, habilidades que les permiten crear un conjunto de hábitos en los cuales han logrado centrar sus metas formativas y de trabajo en una forma más específica que lo que se vive en condiciones de formación presencial.

Ahora la meta corresponde a continuar los seguimientos de egresados no solo a nivel transversal sino que también longitudinal, con el fin de conocer las tendencias que van tomando los papeles laborales que ellos están desarrollando. Por ahora se cuenta con datos descriptivos que puedan permitirnos una dimensión de las condiciones de este sistema, pero será factible recurrir a productos de comparación formal, que superen los niveles exploratorios, y nos brinden mejores explicaciones.

En el marco de contar con una década de formación universitaria en línea, conviene enfatizar sobre la importancia de estas investigaciones a nivel evaluativo. En el presente estudio nos ha permitido la obtención de 
información útil para la posible toma de decisiones del sistema con el único fin real del valor universitario: la formación profesional de alto rigor y calidad educativa.

\section{Referencias}

Carbajal, S. (1998). Seguimiento de egresados de la generacion 88-91 de la licenciatura en psicologia de la ENEP Iztacala. (Tesis de Licenciatura). Recuperado de la Biblioteca Digital de la UNAM.

Dávila, A. P. D (2014). Segundo Informe de Actividades. Pág. 30. Consultado el 23 de junio de 2014 en: http://direccion.iztacala.unam.mx/PDFs/ segundo_informe_PDDA.pdf

Figueroa, S., Bernal, B., \& Andrade, C. (2010). Evaluación de un programa mexicano de maestría en psicología desde la perspectiva del egresado: un estudio sobre los indicadores de calidad. Revista de la Educación Suiperior. 34(1) 23-41

Galindo, Edgar. (2004). Análisis del desarrollo de la psicología en México hasta 1990: Con una bibliografía in extenso. Psicología para América Latina, Recuperado en 07 de octubre de 2014, de http://pepsic.bvsalud.org/scielo.php?script=sci_ arttext\&pid=S1870-350X2004000200004\&Ing=p t\&tIng=es.

Peña C., M. (2009). Formación para la investigación educativa. Una primera mirada a la producción investigativa en los congresos del COMIE. Conferencia presentada en el X Congreso Nacional de Investigación Educativa en Xalapa, Veracruz.

Universidad Autónoma Metropolitana, Unidad Iztapalapa. (2003). Estudio de Seguimiento de Egresados. Generaciones 1998 y 2003. Recuperado en: http://www.egresados.uam.mx/ Est9803/Iztapalapa/CSH/Licenciatura\%20en\%20 Psicologia\%20Social\%201998-2003.pdf 4.0 Internacional, por lo que su contenido gráfico y escrito se puede compartir, copiar y redistribuir total o parcialmente sin necesidad de permiso expreso de su autor con la única condición de que no se puede usar con fines directamente comerciales y los términos legales de cualquier trabajo derivado deben ser los mismos que se expresan en la presente declaración. La única condición es que se cite la fuente con referencia a la Revista Digital Internaciona de Psicología y Ciencia Social y a su autor. 


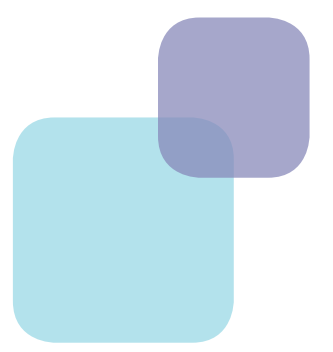

\section{MetA-ANÁlisis del Artículo}

A continuación se muestran los análisis de las puntuaciones otorgadas a este artículo por sus revisores 


\section{Dimensión Cuantitativa}

\section{Perfil de Evaluación entre Pares}
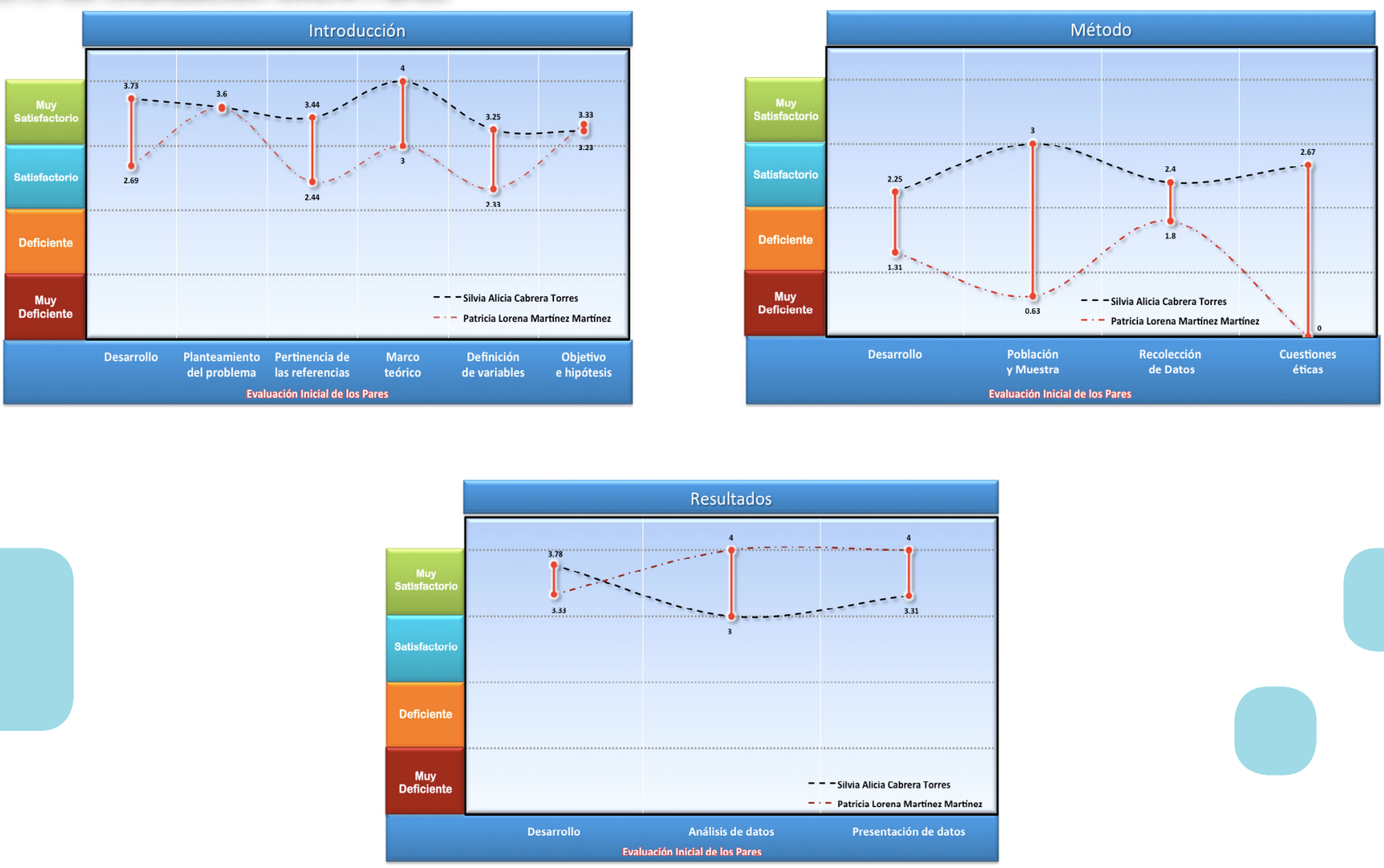

\section{Todos los apartados}

Muy

Satisfactorio

Satisfactoric

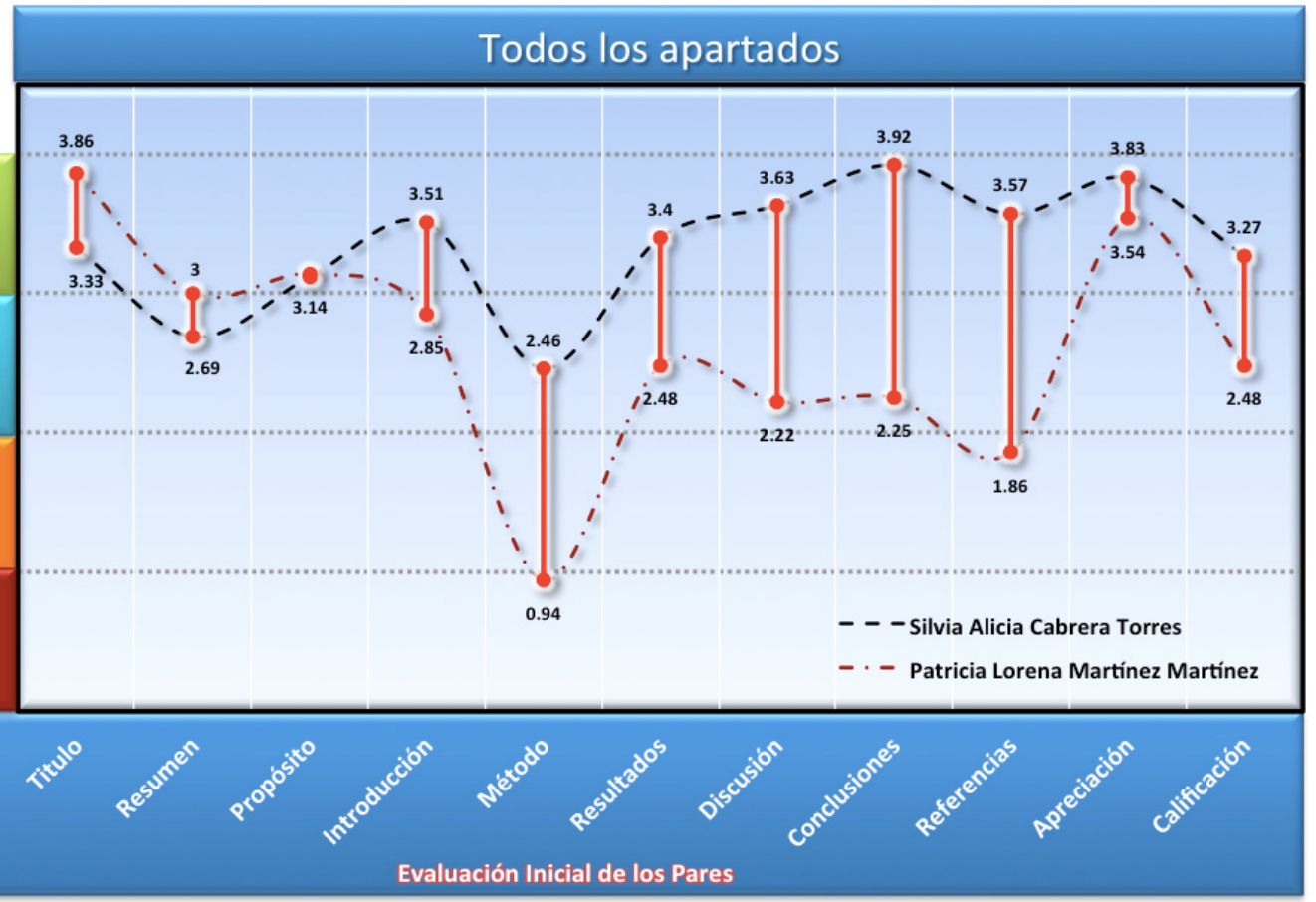

Deficiente

Muy

Deficiente 


\section{Índice de Concordancia}

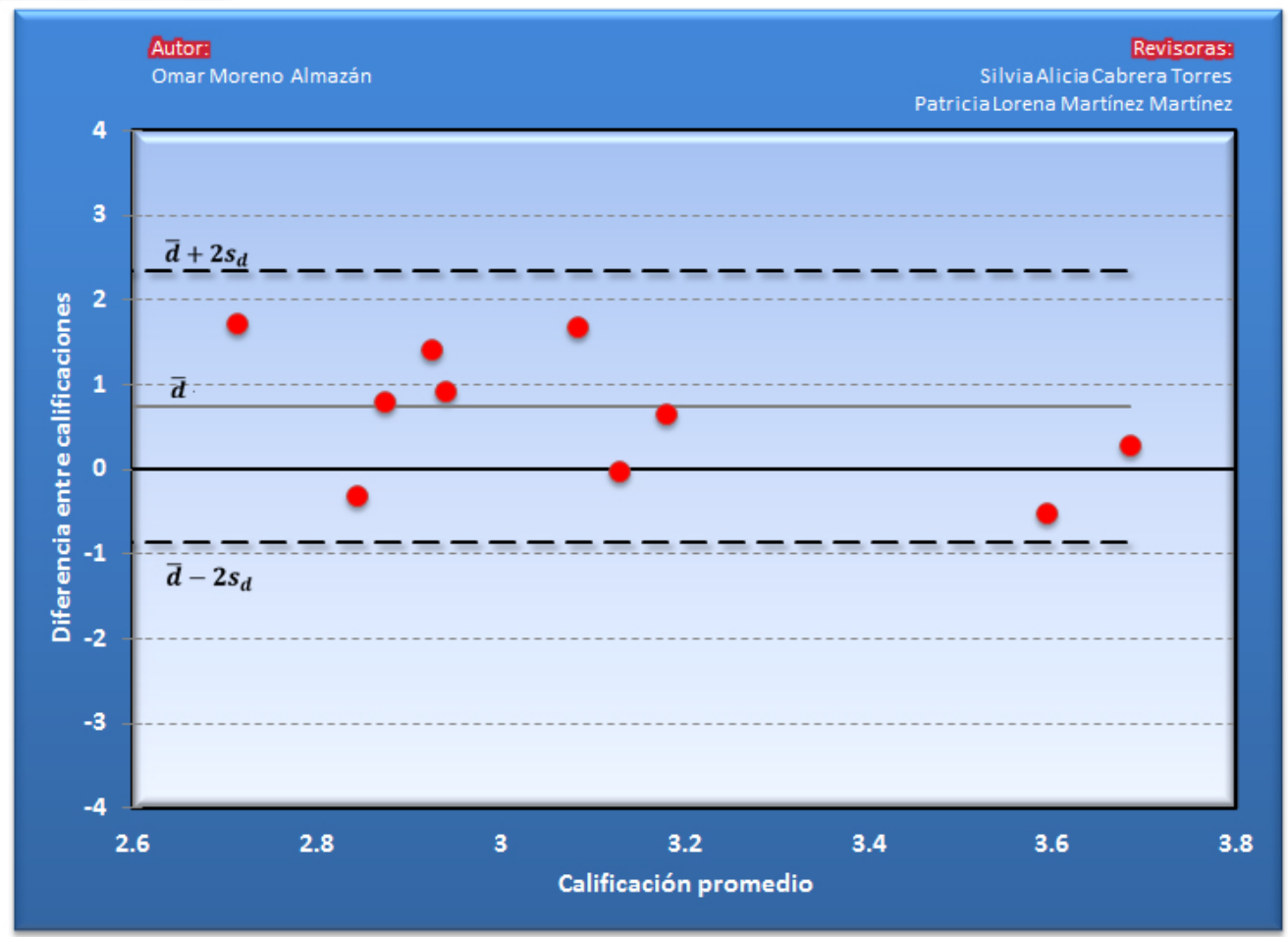

\section{Índice de Acuerdo}

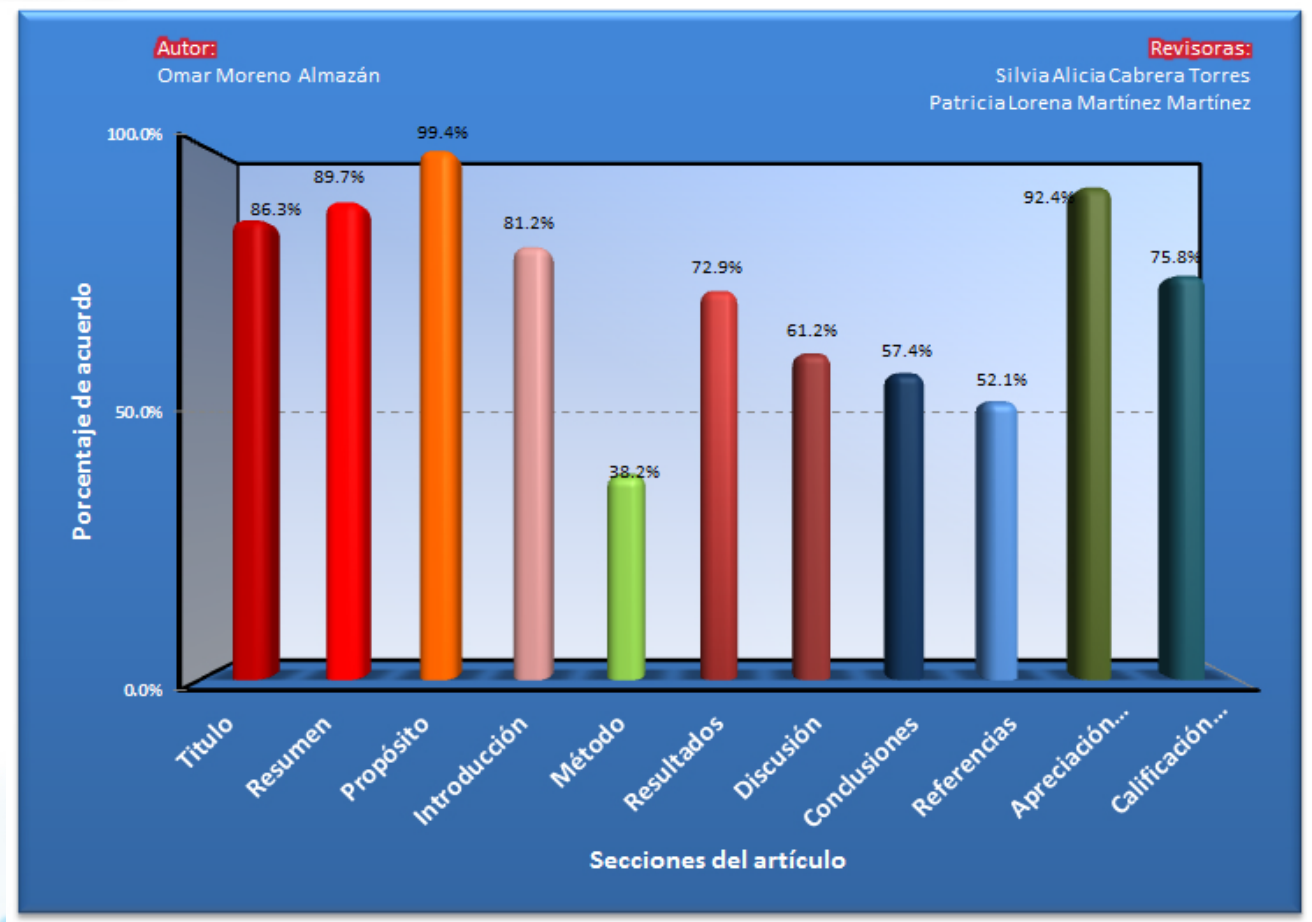




\section{Dimensión Cualitativa}

\begin{tabular}{l|l}
\multicolumn{1}{c|}{ Revisor 1} & \multicolumn{1}{c}{ Revisor 2} \\
\hline Silvia Alicia Cabrera Torres & \multicolumn{1}{c}{ Patricia Lorena Martínez Martínez } \\
\hline Considero bien el título. & $\begin{array}{l}\text { Habría que valorar si deba aparecer la palabra } \\
\text { evaluación para especificar el tipo de estudio. Ejemplo } \\
\text { cambiar en subtítulo a: una evaluación sobre los } \\
\text { egresados. }\end{array}$
\end{tabular}

ResUMEN

No se establece de forma clara la metodología, los resultados y las conclusiones. No está dividido en párrafos y excede las 150 palabras.
En el resumen es importante dejar claramente expuesto el tipo de estudio y el nivel de medición de las variables así como el diseño metodológico.

\section{Próposito del Estudio}

No me quedan claras las preguntas de investigación porque no son mencionadas en el propósito.
Habría que empatar en términos de redacción la pregunta que orienta el inicio de la discusión y el propósito planteado al final de la introducción.

\section{INTRODUCCIÓN}

Mi sugerencia es que se establezca de forma más clara las hipótesis y las variables. No son definidas operacionalmente.
La introducción cuenta con algunos problemas de hilo conductor y redacción que fueron especificados en el archivo adjunto. Es importante que cuanto se cita en cualquier parte del trabajo se reporte en la lista de referencia según lo señale el APA. La operacionalización de las variables a medirse hay que aclarar cómo lograrán medir lo que pretenden, cuántos ítems conforman cada variable, qué tipo de preguntas se formulan (aunque sea ejemplarmente por razones de espacio).

\section{MÉTOdO}

Solo se menciona el instrumento que es el cuestionario de seguimiento a egresados y que es de escala tipo Likert, pero no se menciona los parámetros de la escala. Tampoco se menciona la paquetería utilizada para el cálculo de frecuencias. Considero que falta mencionar la razón por la cual solo se tomaron en cuenta a 34 egresados si se enviaron 50 cuestionarios, así como detallar más el procedimiento. No se menciona si hay consentimiento informado. Falta especificar la validez del instrumento aplicado.
Algunos problemas con la muestra, la selección de egresados, el explicar en qué consiste el instrumento, en ocasiones de la impresión de que asume que quien lee conoce dicho instrumento. Pareciera que el estudio es un procedimiento propio del seguimiento de egresados por lo que da por sentado que el instrumento es el apropiado para indagar lo que se pretende en el estudio. Evitar supuestos y clarificar más en la sección de método e instrumentos así como análisis de datos estadísticos. 


\section{Resultados}

No está clara la pregunta de investigación. No es convincente la presentación de datos, considero que debe existir una comparación en tablas o gráficas de lo mencionado en el marco teórico con los resultados obtenidos, tomando en cuenta que es un estudio que se está realizando por primera vez.
Empatar las preguntas que aparecen en los resultados con lo planteado en la introducción, que estén explícitas desde el inicio un grupo guía de preguntas de investigación. Falta mayor claridad en aspectos como saber quiénes trabajan y en qué concretamente trabajan para saber distinguir los que están ejerciendo de los que no.

\section{Discusión}

Me gustó la apertura que se muestra para ampliar la investigación. Considero que sería importante hacer comparación de las dimensiones 1 y 4 con la modalidad presencial.
La discusión va unida a las conclusiones según lo señala el título, lo cierto es que pareciera que la guía de las mismas es una pregunta (¿cuál es la necesidad conceptual y formativa de crear seguimiento de egresados?) que no empata del todo con el propósito del estudio: el "propósito del presente estudio ha sido describir las condiciones del ejercicio profesional que mantienen los egresados en la carrera de psicología, adscritos al Sistema de Universidad Abierta y Educación a Distancia (SUAED), de la UNAM campus Iztacala, a partir de la exploración de su trayecto laboral y el impacto de la formación recibida durante su licenciatura". Es decir, la meta del estudio era describir las condiciones del ejercicio profesional de los egresados en psicología no tanto argumentar la necesidad conceptual y formativa de que hubiera un seguimiento de egresados, ciertamente vincula esta pregunta con el propósito pero no son lo mismo en cuanto tal. Es pues importante clarificar desde el inicio preguntas de investigación, propósitos del estudio y evidenciar su cumplimiento a lo largo de resultados y discusión.

\section{CONCLUSIONES}

El problema central es que se diluye el problema de investigación en esta parte, apareciendo una pregunta que guía la discusión y conclusiones, lo que podría desviar un tanto la exposición del análisis del resultado a la luz del propósito planteado en la introducción. Insisto en aclarar las preguntas y propósitos del estudio desde inicio y mantenerlos a lo largo de todo el trabajo.

Continúa ... 


\section{RefERENCIAS}

Anexaría mayor bibliografía con respecto a los sistemas a distancia para de igual manera, hacer un comparativo con respecto con lo que se menciona al principio de la introducción con respecto a países desarrollados y su incursión al mundo "virtual".
Revisar que material citado esté referenciado y viceversa. Hay autores citados que no aparecen en esta lista, ojo, es importante revisarla, a su vez hay autores que aparecen en esta lista y no fueron citados. También necesita tomar en cuenta las demandas del APA en las referencias de fuentes como tesis no publicadas, la manera de reportar los datos del o los autores, que haya coincidencia entre siglas usadas para autores institucionales dentro del texto y en la lista de referencias. Percibo una lista de referencias pobre y además descuidada en su formato.

Impresión General del MANuscrito

Solo observé dos errores ortográficos en las páginas 3 y 19 y la repetición de una palabra en la página 20.
En el trabajo se detectaron algunos problemas de redacción principalmente monotonía (repetición reiterada de una misma palabra o expresión gramatical), cacofonía (repetición muy cercana de una misma sílaba, lo cual es válido en el contexto de la poseía), solecismo (en base a....) y algunos problemas de hilo conductor que fueron señalados en el propio trabajo que se enviaré en adjunto.

COMENTARIO POR DICTAMEN

Considero que es un buen tema de investigación y como lo menciona, no hay investigaciones en México en cuanto a esta temática. Sugiero que se comparen los resultados con otros obtenidos en investigaciones previas para licenciatura en modo presencial para poder establecer un parámetro de comparación.
Aceptarlo al incorporar correcciones solicitadas, tiene el trabajo posibilidades de pulirse, si logra justificar aspectos como la cantidad de participantes seleccionados, las razones del instrumento, añadir preguntas de investigación empatándolas con el propósito del estudio, podría ser un buen trabajo. Si se documenta con fuentes teóricas más actuales, si completa lo del método y empata resultados, discusión y conclusiones con lógica en su despliegue podría ser un buen trabajo. Esto además de pulir en lo general la redacción en los aspectos señalados. 


\section{IR A LA HISTORIA DEL Proceso EDITORIAL}


